UNIVERSIDADE DE SÃO PAULO

ESCOLA DE ENFERMAGEM

SILVIA MARIA RIBEIRO OYAMA

\title{
A TEORIA COGNITIVA COMPORTAMENTAL NA INTERVENÇÃO TELEFÔNICA PARA CESSAÇÃO DO TABAGISMO
}

SÃO PAULO

2011 
SILVIA MARIA RIBEIRO OYAMA

\section{A TEORIA COGNITIVA COMPORTAMENTAL NA INTERVENÇÃO TELEFÔNICA PARA CESSAÇÃO DO TABAGISMO}

Tese apresentada à Escola de Enfermagem da Universidade de São Paulo para obtenção do título de Doutor em Ciências.

Área de Concentração:

Enfermagem na saúde do adulto e do idoso

Orientadora: Prof ${ }^{a}$. $\mathrm{Dr}^{\mathrm{a}}$. Eliane Corrêa Chaves

SÃO PAULO

2011 
AUTORIZO A REPRODUÇÃO E DIVULGAÇÃO TOTAL OU PARCIAL DESTE TRABALHO, POR QUALQUER MEIO CONVENCIONAL OU ELETRÔNICO, PARA FINS DE ESTUDO E PESQUISA, DESDE QUE CITADA A FONTE.

Assinatura:

Data:

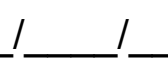

Catalogação na Publicação (CIP)

Biblioteca "Wanda de Aguiar Horta"

Escola de Enfermagem da Universidade de São Paulo

Oyama, Silvia Maria Ribeiro

A Teoria Cognitiva Comportamental na intervenção telefônica para cessação do tabagismo / Silvia Maria Ribeiro Oyama. - São Paulo, 2011.

$143 \mathrm{p}$.

Tese (Doutorado) - Escola de Enfermagem da Universidade de São Paulo.

1. Tabagismo 2. Telefone 3. Terapia Cognitiva 4. Terapia Comportamental I. Título 
Nome: Silvia Maria Ribeiro Oyama

Título: A Teoria Cognitiva Comportamental na Intervenção telefônica para cessação do tabagismo.

Tese apresentada à Escola de Enfermagem da Universidade de São Paulo para obtenção do título de Doutor em Ciências.

Aprovado em _ _ I I

Banca Examinadora

Prof. Dr. Instituição

Julgamento Assinatura

Prof. Dr. Instituição Julgamento Assinatura

Prof. Dr. Instituição Julgamento Assinatura

Prof. Dr. Julgamento Instituição Assinatura 


\section{DEDICATÓRIA}

A Deus, porque permitiu que este trabalho acontecesse.

Ao meu marido, Márcio Andrade pela paciência, amor incondicional, apoio e carinho, tornando-se meu porto seguro.

A minha família, por acreditar e apostar em meus ideais. 


\section{AGRADECIMENTOS}

A Profa Dra. Eliane Corrêa Chaves por me guiar e me ensinar a arte de "Ser Humano" e de "Ser Enfermeira", obrigada por acreditar em mim até mesmo nos momentos que nem eu acreditava. Você é meu exemplo, a profissional que um dia almejo ser semelhante.

Aos amigos Alfredo Pina, Sheila Cozin, Larissa Naves, Yara Ribeiro pela amizade, apoio e convívio saudável

As instituições Faculdade Campo Limpo Paulista e Centro Universitário Padre Anchieta, em especial aos coordenadores Elaine Crepald e Cristiano Mendes pela oportunidade e confiança.

As funcionárias da pós graduação e biblioteca da EEUSP.

Aos participantes deste estudo por acreditar em meu trabalho.

Aqueles que direta ou indiretamente permitiram que esse trabalho se concretizasse. 
Oyama SMR. A Teoria Cognitiva Comportamental na intervenção telefônica para cessação do tabagismo [tese]. São Paulo: Escola de Enfermagem, Universidade de São Paulo; 2011.

\section{RESUMO}

A Teoria Cognitiva Comportamental (TCC) tem sido utilizada nos programas de cessação do tabagismo e tem apresentado resultados satisfatórios na sua aplicação. A abordagem telefônica também tem sido utilizada no tratamento dos fumantes, seu uso é recente e sua efetividade ainda não é consolidada. No Brasil, não existe um protocolo para a abordagem telefônica do fumante estruturado na TCC, assim este estudo teve como objetivo verificar a efetividade de um protocolo para cessação do tabagismo baseado na utilização de ambos. Para isso o perfil tabagístico e a dependência a nicotina foram avaliadas através da aplicação de questionário após o tempo controle, a intervenção e após seis meses do término da abordagem. Foram incluídos 101 indivíduos integrantes de uma UBS no estado de São Paulo. Cada indivíduo recebeu oito contatos com duração média de quarenta minutos, onde foi abordada a identificação e análise dos pensamentos automáticos, crenças disfuncionais, análise funcional do comportamental e emprego de técnicas para mudança de comportamento. Obteve-se uma abstinência imediata de $67,3 \%(p=0,001)$ e após seis meses, $37,6 \%$ de abstinência. Observou-se diminuição significativa dos cigarros fumados por dia $(p=0,001)$ e diminuição da dependência à nicotina avaliado através do teste de dependência à nicotina, teste Fargeström, $(p=0,001)$. Houve associação entre o sexo dos participantes e o resultado do estudo. Estes resultados sugerem uma efetividade do uso do protocolo baseado na Teoria Cognitiva Comportamental na intervenção telefônica para cessação do tabagismo na amostra estudada.

PALAVRAS CHAVE: Tabagismo, Telefone, Terapia Cognitiva, Terapia Comportamental. 
Oyama SMR. The Cognitive Behavioral Theory in the telephone intervention for smoking cessation [Thesis]. São Paulo (SP) Brasil: Escola de Enfermagem, Universidade de São Paulo; 2011.

\begin{abstract}
ABSTRTACT
Cognitive Behavioral Theory (CBT) has been used in programs for stopping smoking and has shown satisfactory results in their application. The telephone approach has also been used in the access to smokers, this use is recent and its effectiveness is not yet consolidated. In Brazil, there is no record for dealing in telephone approach to smoker (CBT), so this study is aimed to verify the effectiveness for stopping smoking based on the use of both. Therefore, the profile of the tobacco user and nicotine dependence were assessed using a questionnaire after the time control, intervention and six months after the end of the approach. 101 members of UBS in the state of Sao Paulo were enrolled in the program. Each person received eight contacts with an average duration of forty minutes, which dealt with the identification and analysis of automatic thoughts, dysfunctional beliefs, functional behavior analysis and use of techniques to change behavior. Results showed an immediate withdrawal of $67.3 \%(p=0.001)$ and $37.6 \%$ after six months of abstinence. We observed a significant reduction in cigarettes smoked per day $(p=0.001)$ and decreased nicotine dependence measured by the test of nicotine dependence, Fargeström test $(p=0.001)$. There has been an association between sex of participants and the study's outcome. These results suggest effectiveness of using a protocol based on Cognitive Behavioral Theory in telephone intervention for stopping smoking in this population.
\end{abstract}

KEYWORDS: Smoking. Phone. Cognitive Therapy. Behavioral Therapy. 


\section{LISTA DE ILUSTRAÇÕES}

Figura 1- Teoria Cognitiva 28

Figura 2- Distribuição temporal dos 40 atendimentos telefônicos

Figura 3- Distribuição temporal do contato final $\quad 40$

Figura 4- Questionamento dos pensamentos 48 automáticos

Figura 5- Modelo de construção da 49 conceituação

Figura 6- $\quad$ Análise da conceituação cognitiva e 50 dos pensamentos automáticos de um dos participantes

Figura 7- Diário de um cliente do programa de 52 intervenção telefônica

Figura 8- Distribuição dos indivíduos conforme 67 o tempo que fuma

Figura 9- Distribuição do grupo nos tempos 70 estudados, conforme quantidade de cigarros fumados por dia nos tempos T0, T1, T2

Figura 10- Distribuição do grupo nos tempos estudados, conforme as quantidades de tentativas anteriores de cessação do tabagismo 


\section{LISTA DE TABELAS}

Tabela 1- Distribuição do grupo nos tempos estudados, conforme quantidade de cigarros fumados por dia

Tabela 2- Distribuição do grupo segundo o tempo estudado, conforme a redução do número de cigarros fumados por dia

Tabela 3- Distribuição do grupo nos tempos estudados, conforme a cessação do tabagismo

Tabela 4- Distribuição do grupo nos tempos estudados, conforme o resultado do teste dependência à nicotina

Tabela 5- Distribuição do grupo nos tempos estudados conforme pergunta 1 do Teste de Fagerström

Tabela 6- Distribuição do grupo nos tempos estudados conforme pergunta 2 do Teste de Fagerström, Mairiporã

Tabela 7- Distribuição do grupo nos tempos estudados, conforme pergunta 3 do Teste de Fagerström

Tabela 8- Distribuição do grupo nos tempos estudados, conforme pergunta 4 do Teste de Fagerström

Tabela 9- Distribuição do grupo nos tempos estudados, conforme pergunta 5 do Teste de Fagerström

Tabela 10- Distribuição do grupo nos tempos estudados, conforme pergunta 6 do Teste de Fagerström

Tabela 11- Distribuição dos indivíduos que pararam de fumar após a intervenção nos tempos estudados, conforme a redução de cigarros fumados por dia 
Tabela 12- Distribuição dos indivíduos que pararam de fumar após a intervenção nos tempos estudados, conforme o resultado do teste dependência à nicotina

Tabela 13- Distribuição dos indivíduos que pararam de fumar após a intervenção nos tempos estudados, conforme pergunta 1 do Teste de Fagerström

Tabela 14- Distribuição dos indivíduos que pararam de fumar após a intervenção nos tempos estudados, conforme pergunta 2 do Teste de Fagerström

Tabela 15- Distribuição dos indivíduos que pararam de fumar após a intervenção nos tempos estudados, conforme pergunta 3 do Teste de Fagerström

Tabela 16- Distribuição dos indivíduos que pararam de fumar após a intervenção nos tempos estudados, conforme pergunta 4 do Teste de Fagerström

Tabela 17- Distribuição dos indivíduos que pararam de fumar após a intervenção nos tempos estudados, conforme pergunta 5 do Teste de Fagerström

Tabela 18- Distribuição dos indivíduos que pararam de fumar após a 82 intervenção nos tempos estudados, conforme pergunta 6 do Teste de Fagerström

Tabela 19- Distribuição do grupo nos tempos estudados, conforme presença de tentativa anterior de cessação do tabagismo 
Tabela 20- Distribuição do grupo nos tempos estudados, conforme quantidade

de tentativas anteriores de cessação do tabagismo

Tabela 21- Distribuição do grupo nos tempos estudados, conforme presença de motivo, exceto problema de saúde, que levou o indivíduo a tentar parar de fumar

Tabela 22- Associação no grupo de estudo das características sócio-

demográficas com cessação do tabagismo e com resultado do teste Fagerström

Tabela 23- Tabela 23- Associação no grupo estudado das características do fumante e cessação do tabagismo

Tabela 24- Associação no grupo estudado das perguntas do teste de Fagerström com cessação do tabagismo 


\section{LISTA DE ABREVIATURAS E SIGLAS}

$\begin{array}{ll}\text { CPS } & \text { Centro de Promoção da Saúde } \\ \text { EI } & \text { Estímulo Incondicionado } \\ \text { EUA } & \text { Estados Unidos da América } \\ \text { IBGE } & \text { Instituto Brasileiro Geografia e Estatística } \\ \text { INCA } & \text { Instituto Nacional do Câncer } \\ \text { R } & \text { Resposta } \\ \text { S } & \text { Estímulos } \\ \text { SNC } & \text { Sistema Nervoso Central } \\ \text { TCC } & \text { Teoria Cognitiva Comportamental } \\ \text { TOFHLA } & \text { Teste de Alfabetização funcional para } \\ & \text { adultos na área de saúde } \\ \text { TRN } & \text { Terapia de Reposição de Nicotina } \\ \text { T0 } & \text { Tempo 0 } \\ \text { T1 } & \text { Tempo 1 } \\ \text { T2 } & \text { Tempo 2 } \\ \text { T3 } & \text { Tempo 3 } \\ \text { UBS } & \text { Unidade Básica de Saúde } \\ \text { VIGITEL } & \text { Sistema de Vigilância de Fatores de Risco e } \\ & \text { Proteção para Doenças Crônicas por } \\ & \text { Inquérito Telefônico }\end{array}$

